\title{
Prognostic value of Dickkopf-1 and ß- catenin expression in advanced gastric cancer
}

\author{
Soon Auck Hong ${ }^{1}$, Su Hyun Yoo ${ }^{2}$, Han Hong Lee ${ }^{3}$, Der Sheng Sun ${ }^{4}$, Hye Sung Won ${ }^{4}$, Okran Kim \\ and Yoon $\mathrm{Ho} \mathrm{Ko}^{4,5^{*}}$
}

\begin{abstract}
Background: Dickkopf-1 (DKK1) is a Wnt/ß-catenin pathway antagonist related to gastric cancer (GC) carcinogenesis. However, the prognostic role of combined DKK1 and B-catenin expression in advanced GC (AGC) is not clear.

Methods: In total, 158 patients with AGC who underwent gastric resection were enrolled in this study. DKK1 and B-catenin expression was evaluated in whole tumor sections by immunohistochemistry.

Results: DKK1 expression was high in 73 (46.2\%) patients, while ß-catenin expression was positive in 51 (32.3\%) patients. The expression of DKK1 was positively correlated with that of $\beta$-catenin $(P<0.001)$. The combined expression of DKK1 and B-catenin was significantly associated with high N stage (N2 and N3) ( $P=0.042$ ). In addition, patients with high DKK expression demonstrated poorer overall (OS) $(P<0.001)$ and disease-free survival (DFS) $(P=0.001)$. However, there were no differences between high DKK1 expression with $B$-catenin positivity and high DKK1 expression with $B$-catenin negativity (OS, $P=0.379$ : DFS, $P=0.255)$. Multivariate analysis revealed that high DKK1 alone or high DKK1 with B-catenin positivity were independent prognostic factors for both OS (high DKK1: hazard ratio [HR], 2.130; 95\% confidence interval [Cl]; 1.370-3.312, $P=0.001$; high DKK1 with B-catenin positivity: HR, 2.140; 95\% Cl, 1.343-3.409: $P=0.001$ ) and DFS (high DKK1: HR, 2.092; 95\% Cl, 1.180-3.708; $P=0.012$; high DKK1 with B-catenin positivity: HR, 2.357; $95 \% \mathrm{Cl}, 1.291-4.306 ; P=0.005)$.
\end{abstract}

Conclusion: Our results indicate that high DKK1 expression regardless of ß-catenin positivity is a crucial prognostic factor for predicting tumor recurrence and survival in patients with resected AGC.

Keywords: Dickkopf-1, Gastric cancer, Prognosis, Cut value

\section{Background}

Gastric cancer is the fourth most commonly diagnosed cancer and the second most common cause of cancerrelated death worldwide [1]. Recently, the development of surgical techniques and target therapies has led to a significant improvement in survival. Currently, early gastric carcinoma (EGC) has a 5-year survival rate of over 90\% [2]. However, for cases of advanced GC (AGC), the

\footnotetext{
* Correspondence: koyoonho@catholic.ac.kr

${ }^{4}$ Division of Oncology, Department of Internal Medicine, College of Medicine, The Catholic University of Korea, 222, Banpodaero, Seochogu, Seoul 06591, Republic of Korea

${ }^{5}$ Cancer Research Institute, College of Medicine, The Catholic University of Korea, Seoul, Republic of Korea

Full list of author information is available at the end of the article
}

survival rate is still around 40\% [3]. Thus, a new biomarker for AGC is urgently needed.

The Wnt signaling cascade governs cell proliferation, cell polarity, and cell fate during embryonic development and homeostasis in human tissues [4]. Solid tumors frequently exhibit dysregulated Wnt signaling pathways, and this dysregulation is linked to enhanced malignant potential [5]. In $\mathrm{GC}$, activation of the $\mathrm{Wnt} / \mathrm{\beta}$-catenin pathway is found in approximately $30 \%$ to $50 \%$ of tumors [6, 7]. The first step in activation is the binding of the Wnt ligand to the sevenpass transmembrane Frizzled receptor (FZ), low-density lipoprotein receptor-related protein 6 (LRP 6), and LRP 5. The Wnt-FZ-LRP5/6 complex disrupts Axin-mediated Bcatenin phosphorylation, resulting in $\beta$-catenin stabilization. Accumulation of $B$-catenin in the cytoplasm results in its

(c) The Author(s). 2018 Open Access This article is distributed under the terms of the Creative Commons Attribution 4.0 International License (http://creativecommons.org/licenses/by/4.0/), which permits unrestricted use, distribution, and 
transport to the nucleus, where $ß$-catenin forms complexes with T-cell factor (TCF)/lymphoid enhancer factor and activates Wnt-targeted gene expression (cyclin D1, $c-M Y C$, axin-2) $[5,8]$. Wnt signaling can be repressed by six Wnt antagonist families, including Dickkopf (DKK) proteins, secreted Frizzled-related proteins, WNT-inhibitory factor 1, Wise/SOST, Cerberus, and insulin-like growth-factor binding protein 4 [9]. DKK1, the most well-known Wnt antagonist, is a $35 \mathrm{kDa}$ protein that contains a secreted signal peptide sequence [10]. Antagonism of the Wnt pathway via antagonists such as DKK1 is accomplished through binding to LRP 5/6 [11, 12]. Despite being a negative regulator of Wnt signaling, the prognostic role of DKK1 is not well understood. Results from previous studies on DKK1 in diverse tumors have demonstrated conflicting results; some have reported that DKK1 acts as a tumor suppressor, while others have shown that it acts as an oncogene [13]. Lee et al. and Gao et al. reported that overexpression of DKK1 protein and mRNA in tissue and increased levels of DKK1 in serum were significantly associated with unfavorable prognosis in patients with GC $[14,15]$. In contrast, other recent studies showed low DKK1 expression in GC samples, and that restoration of DKK1 in tumor cells inhibited tumor cell growth and invasion [16, 17]. However, the prognostic value of DKK1 in GC is not clearly determined. Furthermore, although DKK1 interacts with $ß$-catenin, the association between DKK1 and abnormal $ß$-catenin expression has not been well studied. Therefore, we aimed to confirm the role of DKK1 as a prognostic factor in AGC and to determine the association between DKK1 and $\beta$-catenin in a sizable AGC cohort using immunohistochemistry.

\section{Methods}

\section{Study population}

The clinical and pathological data of 158 patients with AGC who had undergone primary radical resection between 2001 and 2005 at Uijeongbu St. Mary's Hospital of the Catholic University of Korea were reviewed. The inclusion criteria were (i) pathologically confirmed adenocarcinoma, (ii) radical resection without preoperative radiation or chemotherapy, (iii) removal of at least 15 or more lymph nodes, and (iv) available paraffinembedded tumor specimens. Postoperative pathological staging was based on the American Joint Committee on Cancer (AJCC) staging criteria, 7th edition. This study was approved by the Institutional Research Ethics Board of Uijeongbu St. Mary's Hospital of the Catholic University of Korea and adhered to the Declaration of Helsinki. Patient anonymity was preserved.

\section{Immunohistochemistry}

Immunohistochemistry was performed on formalin-fixed paraffin-embedded tissue sections. Whole tissue sections of representative tumor samples were deparaffinized using xylene and graded alcohol and then rehydrated with distilled water. Blocking of endogenous peroxidase activity was achieved by quenching with 3\% hydrogen peroxide in methanol for $10 \mathrm{~min}$. Antigen retrieval was then performed by heating the slides for $15 \mathrm{~min}$ in 0 . $01 \mathrm{M}$ citrate buffer ( $\mathrm{pH}$ 6.0). The sections were incubated with human specific antibodies against DKK1 (1: 200, Abcam, Cambridge, UK) and ß-catenin (1:100, Cell Signaling, Danvers, MA, USA) at room temperature for $30 \mathrm{~min}$, washed in phosphate-buffered saline, and then incubated with a peroxidase-labeled polymer conjugated to the secondary antibody for $30 \mathrm{~min}$. The immunoreaction was visualized using the Polink-2 plus polymer HRP detection system (Golden Bridge International, Mukilteo, WA, USA). In the negative controls, the primary antibodies were substituted with normal rabbit or mouse IgG at the same concentration as the primary antibody. Immunohistochemical staining was independently examined by two board-certified pathologists (S.A.H. and S.H. Y.) who were blinded to the clinical data. The intensity of DKK1 staining was scored using the following scale: 0 , no staining; 1 , weakly positive; 2 , moderately positive; 3 , strongly positive. The proportion of DKK1 staining was calculated as the percentage of positive tumor cells. $\mathrm{H}$ scores, ranging from 0 to 300 , were determined by multiplying the intensity score by the proportion. The cut-off $\mathrm{H}$ score for high DKK1 expression was set at 60 using maximally selected rank statistics (maxstat) calculated in $\mathrm{R}$ statistical programming language version 3.2.3 (https://www.r-project.org/). B-catenin expression was considered positive if more than $10 \%$ of tumor cells showed nuclear and cytoplasmic staining [18].

\section{Statistical methods}

Chi-square or Fisher's exact tests were conducted to determine the association among DKK1 expression, $\beta-$ catenin expression, and clinicopathologic parameters. A $P$-value of $<0.05$ was considered statistically significant. Overall survival (OS) was calculated from the date of diagnosis to the date of death due to any cause or the last follow-up visit. Disease-free survival (DFS) was calculated from the date of diagnosis to the date of the first distant or local disease recurrence or last follow-up. The Kaplan-Meier method was used to analyze 'time-to-event' data, and statistical differences in the cumulative survival curves were evaluated using the log-rank test. Cox proportional hazards regression models were used to investigate the significance of the prognostic factors. All variables with a $P$-value $<0.05$ in the univariate analysis were included in the multivariate analysis. Survival rates and hazard ratios are shown with their respective 95\% confidence intervals (CIs). Statistical analyses were performed using $\mathrm{R}$ statistical programming language version 3.2. 
3 (https://www.r-project.org) and the SPSS software package (version 18.0; SPSS, Chicago, IL, USA).

\section{Results \\ Patients}

Patient age at diagnosis ranged from 29 to 89 (median: 62) years. The patient cohort consisted of 113 males and 45 females. Based on the AJCC staging criteria, 18 patients (11.4\%) had stage I disease, 50 (31.6\%) had stage II disease, $88(55.7 \%)$ had stage III disease, and $2(1.3 \%)$ had stage IV disease. Ninety-seven (61.4\%) patients received 5-fluorouracil and cisplatin combination therapy postoperatively. The follow-up period ranged from 0 to 152.0 months, with a median of 68.0 months, after curative surgical resection. Of the 158 total patients, 91 (57.6\%) died, and $67(42.4 \%)$ were alive at the last follow-up. Disease recurrence was observed in 52 cases (32.9\%).

\section{DKK1 and B-catenin expression in advanced GC}

In non-neoplastic gastric epithelium, only scant or weak cytoplasmic staining of DKK1 was detected (Fig. 1a), while $ß$-catenin expression was identified as membranous (Fig. 1b). Increased DKK1 expression $\mathrm{H}$ score was found in higher nodal-stage tumors $(\mathrm{N} 0 / 1,61.72 \pm 51.80$ [mean \pm standard deviation] vs. N2/3, $84.79 \pm 70.81$; $P=0.019$ ) (Fig. 2). Based on the determined cut-off $\mathrm{H}$ score for DKK1 (>60), $73(46.2 \%)$ and $85(53.8 \%)$ patients were classified into high and low expression groups, respectively (Fig. 3a and b). High expression of DKK1 was statistically associated only with advanced nodal stage (N0/1 vs. N2/3, $P=0.021$ ) (Table 1 ). $B$-catenin positivity was observed in 51 cases $(32.3 \%)$ and negativity in 107 cases (67.7\%) (Fig. 3c and d). However, ß-catenin positivity was not related to any clinicopathologic factors (Additional file 1: Table S1). A positive correlation between DKK1 and $\beta$-catenin expression was identified $(P<0.001)$ (Table 2). Stratification of patients with both DKK1 and $ß$-catenin expression was performed as follows: high DKK1 expression with $ß$-catenin positivity ( $n=35,22.2 \%)$, high DKK1 expression with $\beta$ catenin negativity $(n=38,24.1 \%)$, low DKK1 expression with $ß$-catenin positivity $(n=16,10.1 \%)$, and low DKK1 expression with $\beta$-catenin negativity $(n=69,43.7 \%)$. High DKK1 expression combined with ß-catenin positivity was related to high nodal stage (N0/1 vs. N2/3, $P=0.042$ ) (Table 3).

\section{Prognostic value of DKK1 and ß-catenin expression in advanced GC}

We assessed the correlation among DKK1 expression, B-catenin expression, and prognosis using KaplanMeier curves with a log-rank test. High DKK1 expression was significantly associated with shorter OS $(P<0.001)$ and DFS $(P=0.001)$ (Fig. $4 \mathrm{a}$ and b). Patients positive for $\beta$-catenin expression showed shorter OS $(P=0.047)$, but there were no differences in DFS between patients positive and those negative for $ß$-catenin expression $(P=0.134)$ (Fig. $4 \mathrm{c}$ and $\mathrm{d}$ ). Survival analysis of the four groups was performed based on combined DKK1 and ß-catenin expression statuses (Fig. 4e and f). Patients with high DKK1 and ß-catenin positivity demonstrated poorer survival compared with patients with low DKK1 and $ß$-catenin positivity (OS, $P=0.009$; DFS, $P=0.016$ ) and patients with low DKK1 and ß-catenin negativity (OS, $P<0.001$; DFS, $P=0.001$ ). However, among the patients with high DKK1 expression, no differences were found between those positive and those negative for B-catenin (OS, $P=0.379$; DFS, $P=0.255)$. The factors affecting OS and DFS were further analyzed using the Cox proportional hazards regression method. The univariate Cox proportional hazard ratios for OS and DFS were calculated. High DKK1 expression with or without $B$-catenin positivity were significantly related with poorer DFS (HR, 2.497; 95\% $\mathrm{CI}, 1.421-4.386 ; P=0.001$ ) and $\mathrm{OS}$ (hazard ratio [HR], 2.509; 95\% CI, 1.637-3.846; $P$ < 0.001) (Table 4). High DKK1 and ß-catenin positivity was significantly

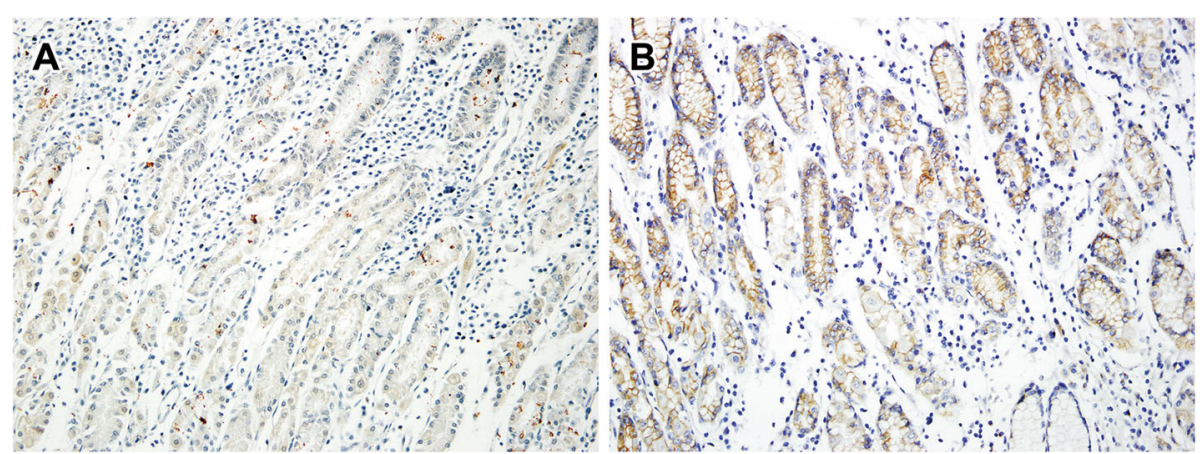

Fig. 1 Immunohistochemical staining of DKK1 and $\beta$-catenin in normal gastric mucosa. Scant expression of DKK1 (a) and membranous staining (b) of $\beta$-catenin were observed 

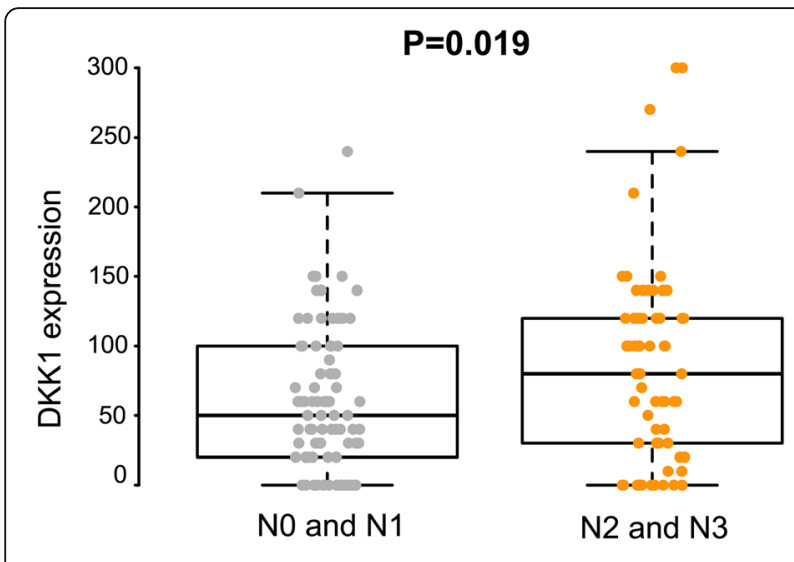

Fig. 2 Expression of DKK1 protein in advanced gastric cancer with $\mathrm{N} 1$ and $\mathrm{N} 2$ is higher than that in N2 and N3

related to unfavorable DFS (HR, 2.512; 95\% CI, 1 . 430-4.413; $P=0.001$ ) and OS (hazard ratio [HR], 2. 522; 95\% CI, 1.645-3.867; $P<0.001$ ) (Table 5) and. Results from the multivariate Cox proportional hazards model showed that high DKK1 expression is an independent prognostic indicator for both DFS (HR, 2.092: 95\% CI, 1.180-3.708: $P=0.012)$ and OS (HR, 2.130: 95\% CI, 1.370-3.312: $P=0.001$ ) (Table 4) and. According to combined DKK1 and $ß$-catenin expression in the multivariate Cox proportional hazard models, high DKK1 and $ß$-catenin positivity is an independent prognostic value for DFS (HR, 2.357; 95\% CI, 1.291-4.306; $P=0.005)$ and OS (HR, 2140; 95\% CI, 1.343-3.409; $P=0.001$ ) (Table 5).

\section{Discussion}

In this study, high DKK1 expression was significantly associated with high $\mathrm{N}$ stage, and patients with high DKK1 expression demonstrated shorter OS and DFS. High DKK1 expression was also correlated with $\beta$-catenin positivity. On multivariate analysis, high DKK1 expression and $\beta$-catenin positivity were found to be independent adverse prognostic factors for both OS and DFS. Interestingly, patients with high DKK1 expression and concomitant $\beta$-catenin negativity also had shorter OS and DFS. Thus, the prognostic value of DKK1 in GC patients may be independent of $\beta$-catenin expression.

Various tumors have shown conflicting results regarding the role of DKK1 as a tumor suppressor or oncogene. DKK1 is a secreted protein that plays a crucial role as a negative regulator of the Wnt signaling pathway, and downregulation of DKK1 in colon cancer, breast cancer, hepatocellular carcinoma, and renal cell carcinoma suggests that DKK1 is an antagonist of the Wnt signaling pathway. However, overexpression of DKK1 has been observed in various malignant tumors and is also correlated with adverse prognosis in patients with multiple myeloma, hepatoblastoma, Wilm's tumor, lung cancer, and breast cancer [19-22].

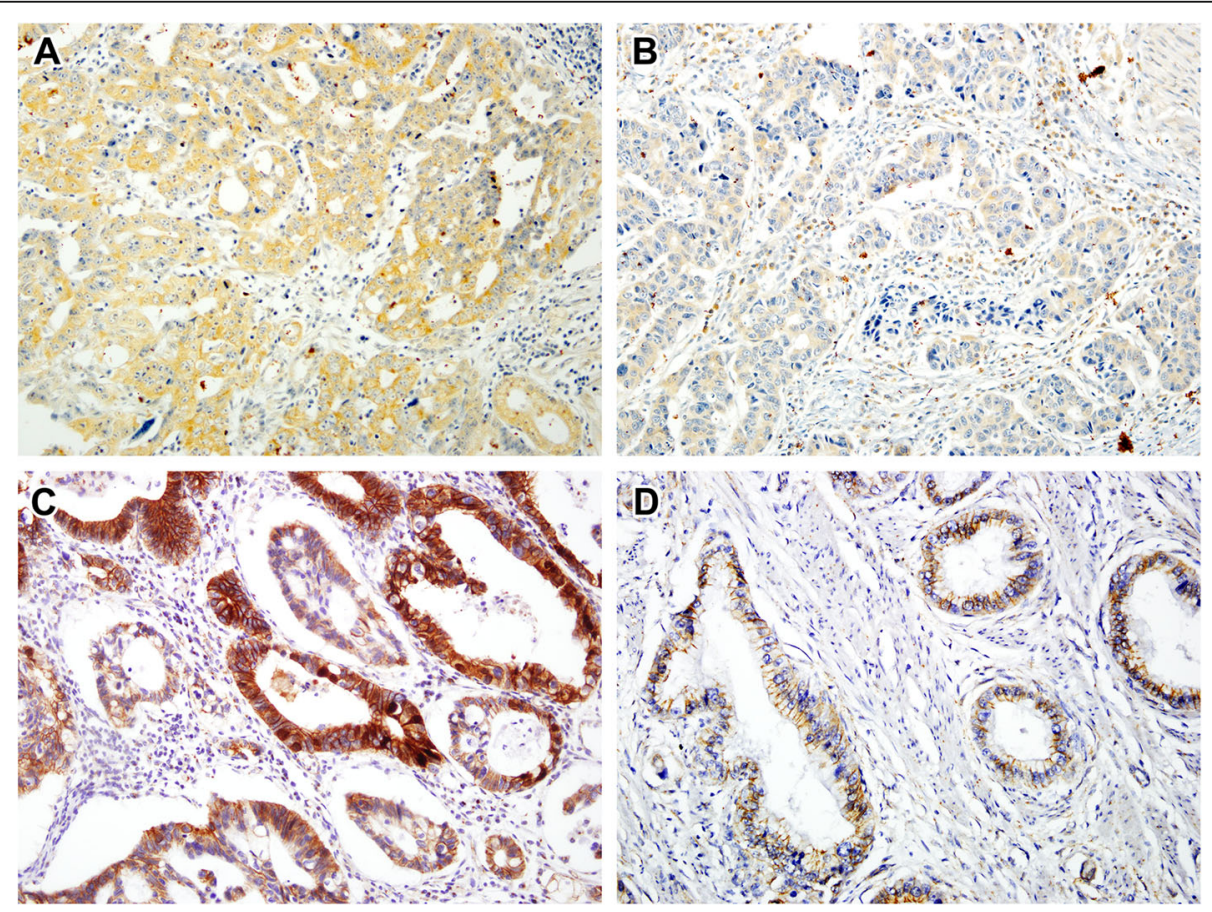

Fig. 3 Immunohistochemical staining of DKK1 and $\beta$-catenin staining in GC. Representative images of GC with high expression of DKK1 (a), low expression of DKK1 (b), positive $\beta$-catenin staining (c), and negative $\beta$-catenin staining (d) 
Table 1 Correlation between clinicopathologic findings and DKK1 expression

\begin{tabular}{|c|c|c|c|c|c|c|c|c|}
\hline \multirow{2}{*}{$\begin{array}{l}\text { Clinicopathologic } \\
\text { features }\end{array}$} & \multirow{2}{*}{$\begin{array}{l}\text { No. of } \\
\text { patients } \\
(\%)\end{array}$} & \multicolumn{2}{|c|}{ DKK-1 expression } & \multirow[t]{2}{*}{$P$-value } & \multirow{2}{*}{$\begin{array}{l}\text { Clinicopathologic } \\
\text { features }\end{array}$} & \multicolumn{2}{|c|}{ High DKK1 and positive ß-catenin } & \multirow[t]{2}{*}{$P$-value } \\
\hline & & $\begin{array}{l}\text { Low (\%) } \\
85(53.8)\end{array}$ & $\begin{array}{l}\text { High (\%) } \\
73(46.2)\end{array}$ & & & $\begin{array}{l}\text { Yes (\%) } \\
35(22.2)\end{array}$ & $\begin{array}{l}\text { No (\%) } \\
123(77.8)\end{array}$ & \\
\hline Age, years & & & & & Age, years & & & \\
\hline$>62$ & $79(50.0)$ & $36(42.4)$ & $43(58.9)$ & 0.038 & $>62$ & $21(60.0)$ & $58(47.2)$ & 0.180 \\
\hline$\leq 62$ & $79(50.0)$ & $49(57.6)$ & $30(41.1)$ & & $\leq 62$ & $14(40.0)$ & $65(52.8)$ & \\
\hline Sex & & & & & Sex & & & \\
\hline Male & $113(71.5)$ & $60(70.6)$ & $53(72.6)$ & 0.780 & Male & $27(77.1)$ & $86(69.9)$ & 0.403 \\
\hline Female & $45(28.5)$ & $25(29.4)$ & $20(27.4)$ & & Female & $8(22.9)$ & $37(30.1)$ & \\
\hline T stage & & & & & T stage & & & \\
\hline $\mathrm{T} 2 / 3$ & $42(26.6)$ & $24(28.2)$ & $18(24.7)$ & 0.612 & $\mathrm{~T} 2 / 3$ & $11(31.4)$ & $31(25.2)$ & 0.462 \\
\hline $\mathrm{T} 4$ & $116(73.4)$ & $61(71.8)$ & $55(75.3)$ & & T4 & $24(68.6)$ & $92(74.8)$ & \\
\hline$N$ stage & & & & & N stage & & & \\
\hline No to N1 & $87(55.1)$ & $54(63.5)$ & $33(45.2)$ & 0.021 & No/1 & $14(40.0)$ & $73(59.3)$ & 0.042 \\
\hline $\mathrm{N} 2$ to N3 & $66(44.9)$ & $31(36.5)$ & $40(54.8)$ & & $\mathrm{N} 2 / 3$ & $21(60.0)$ & $50(40.7)$ & \\
\hline Lauren's classification & & & & & Lauren's classification & & & \\
\hline Diffuse type & $105(66.5)$ & $60(70.6)$ & 45 (61.6) & 0.235 & Diffuse type & $20(57.1)$ & $85(69.1)$ & 0.186 \\
\hline Non-diffuse type & $53(33.5)$ & $25(29.4)$ & $28(38.4)$ & & Non-diffuse type & $15(42.9)$ & 38 (30.9) & \\
\hline Tumor grade & & & & & Tumor grade & & & \\
\hline Well/moderate & $46(29.2)$ & $20(23.5)$ & $26(35.6)$ & 0.095 & Well/moderate & $12(34.3)$ & $34(27.6)$ & 0.445 \\
\hline Poor & $112(71.8)$ & $65(76.5)$ & $47(64.4)$ & & Poor & $23(65.7)$ & $89(72.4)$ & \\
\hline Lymphatic invasion & & & & & Lymphatic invasion & & & \\
\hline Yes & $127(80.4)$ & $65(23.5)$ & $62(84.9)$ & 0.182 & Yes & $28(80.0)$ & $99(80.5)$ & 0.949 \\
\hline No & $31(19.6)$ & $20(76.5)$ & $11(15.1)$ & & No & $7(20.0)$ & $24(19.5)$ & \\
\hline Vascular invasion & & & & & Vascular invasion & & & \\
\hline Yes & $12(7.6)$ & $5(5.9)$ & 7 (9.6) & 0.381 & Yes & $3(8.6)$ & $9(7.3)$ & 0.805 \\
\hline No & $146(92.4)$ & $80(94.1)$ & $66(90.4)$ & & No & $32(91.4)$ & $114(92.7)$ & \\
\hline TNM stage & & & & & TNM stage & & & \\
\hline$|/| \mid$ & $68(43.0)$ & $41(51.8)$ & $27(37.0)$ & 0.155 & $|/| \mid$ & $14(40.0)$ & $54(43.9)$ & 0.681 \\
\hline III/IV & $90(57.0)$ & $44(48.2)$ & $46(63.0)$ & & III/IV & $21(60.0)$ & $69(56.1)$ & \\
\hline
\end{tabular}

Although DKK1 has been investigated extensively in various tumors, including $\mathrm{GC}$, no previous studies have evaluated the association between DKK1 and $\beta$-catenin expression in AGC. Previous studies have shown that overexpression of DKK1 is related to adverse prognosis in $\mathrm{GC}[14,15]$. This is consistent with the results we obtained in the high DKK1 expression group, which showed that regardless of $\beta$-catenin expression, high DKK1 expression is related to unfavorable prognosis. In

Table 2 Correlation between DKK1 and ß-catenin expression

\begin{tabular}{lllll}
\hline Coexpression & & \multicolumn{2}{l}{ DKK1 expression } & \\
\cline { 3 - 4 } & & Low & High & $P$ value \\
\hline B-catenin & Negative & $69(81.2)$ & $38(52.1)$ & $<0.001$ \\
& Positive & $16(18.8)$ & $35(47.9)$ & \\
\hline
\end{tabular}

Table 3 Correlations between clinicopathologic findings and high DKK1 expression with ß-catenin positivity

previous studies, high DKK1 expression was found to be associated with intestinal GC, advanced T stage, vascular and lymphatic invasion, and distant metastasis. However, we only found a significant association between $\mathrm{N}$ stage and high DKK1 expression. This discrepancy might stem from different cut-off values and patient cohorts among studies. Our cut-off value was meticulously determined and optimized for patient survival using maximally selected rank statistics. Therefore, we suggest that our cut-off value better reflects the prognostic role of DKK1 in GC, independently of other adverse clinicopathologic parameters. Previous clinical studies of DKK1 expression in GC included both early GC and AGC, with a number of distant metastasis cases $[14,15]$. Survival of early gastric carcinoma patients is over $90 \%$, but survival of patients with both GC and distant metastasis is less 

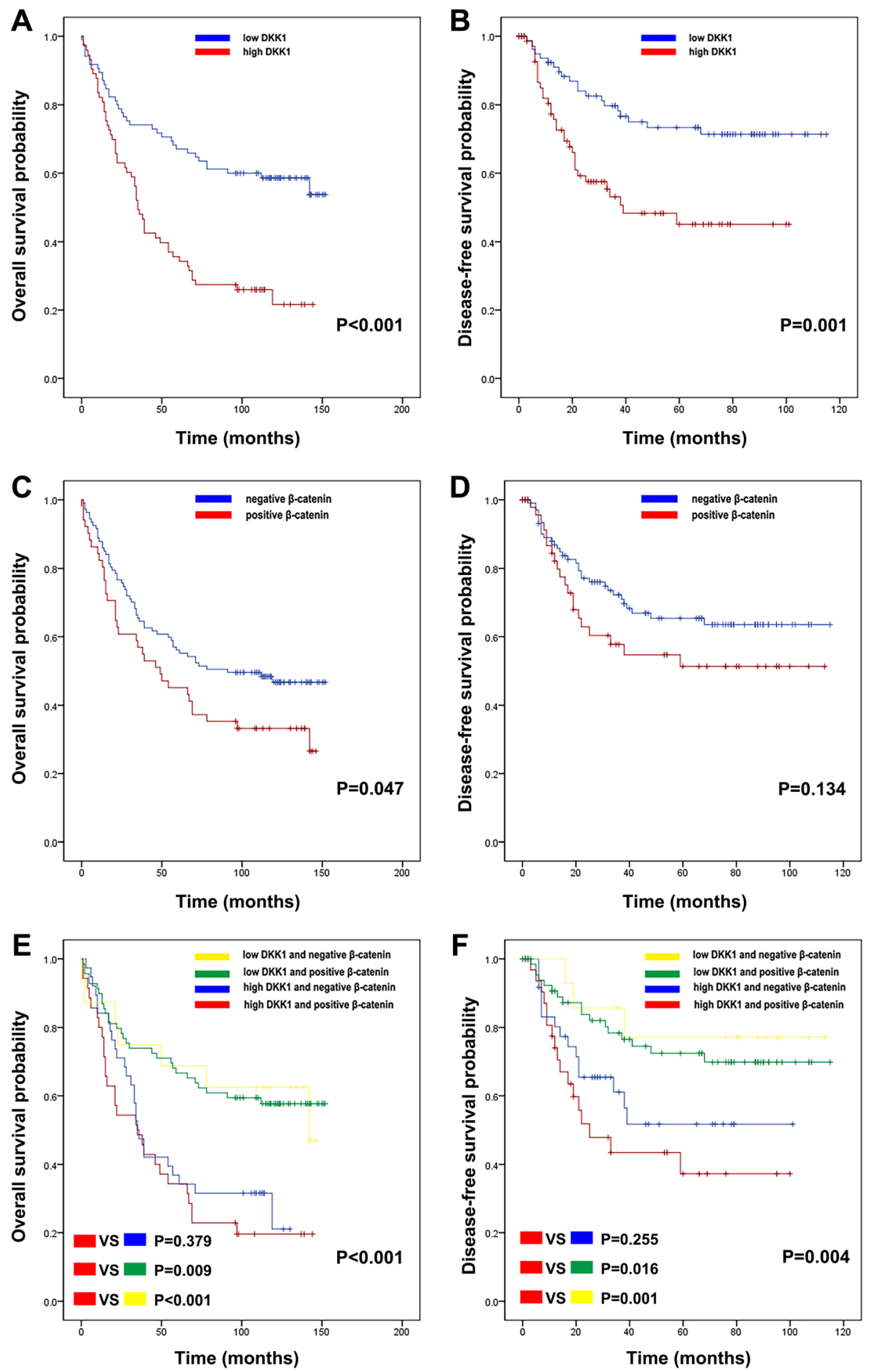

Fig. 4 Kaplan-Meier survival curves were plotted according to DKK1 and $\beta$-catenin protein expression. High DKK1 expression was significantly related to poor OS (a) and DFS (b) in GC patients. Patients with positive $\beta$-catenin staining showed shorter OS (c), while DFS (d) was not different significantly between patients with positive and those with negative $\beta$-catenin staining. The combination of DKK1 and $\beta$-catenin expression was evaluated for its impact on OS (e) and DFS (f)

than $10 \%$ [23]. Therefore, clarification of the prognostic impact of DKK1 could be confounded by the heterogenous patient cohorts used in previous studies.
Wan et al. reported that adenovirus-mediated DKK1 overexpression in $\mathrm{CD} 44^{+} \mathrm{GC}$ cells inhibits tumorigenicity through attenuating Wnt signaling [16]. The results 
Table 4 Univariate and multivariate analyses of disease-free survival and overall survival using the Cox proportional hazards model for all patients

\begin{tabular}{|c|c|c|c|c|c|c|}
\hline \multirow[t]{2}{*}{ Characteristics } & \multicolumn{3}{|c|}{ Univariate analysis of DFS } & \multicolumn{3}{|c|}{ Multivariate analysis of DFS } \\
\hline & Hazard ratio & $95 \% \mathrm{Cl}$ & $p$-value & Hazard ratio & $95 \% \mathrm{Cl}$ & $p$-value \\
\hline Age (> 62 vs. $\leq 62)$ & 1.251 & $0.725-2.158$ & 0.420 & & & \\
\hline Sex (female vs male) & 1.005 & $0.544-1.856$ & 0.987 & & & \\
\hline Advanced T stage (pT4 vs. pT2/3) & 7.564 & $2.356-24.286$ & 0.001 & 6.569 & $2.014-21.422$ & $<0.001$ \\
\hline Advanced N stage (pN2/3 vs. pNo/1) & 3.544 & $1.959-6.409$ & $<0.001$ & 2.500 & $1.359-4.601$ & 0.003 \\
\hline Lauren's classification (diffuse vs. non-diffuse) & 1.255 & $0.680-2.317$ & 0.467 & & & \\
\hline Venous invasion (yes vs. no) & 1.940 & $0.771-4.883$ & 0.159 & & & \\
\hline Tumor grade (poor vs well to moderate) & 1.172 & $0.626-2.197$ & 0.619 & & & \\
\hline Adjuvant chemotherapy (yes vs. no) & 1.196 & $0.687-2.083$ & 0.526 & & & \\
\hline \multirow[t]{11}{*}{ High DKK1 (yes vs. no) } & 2.497 & $1.421-4.386$ & 0.001 & 2.092 & $1.180-3.708$ & 0.012 \\
\hline & \multicolumn{3}{|c|}{ Univariate analysis of OS } & \multicolumn{3}{|c|}{ Multivariate analysis OS } \\
\hline & 1.934 & $1.269-2.946$ & 0.002 & 1.820 & $1.189-2.785$ & 0.006 \\
\hline & 0.656 & $0.403-1.069$ & 0.091 & & & \\
\hline & 3.356 & $1.825-6.170$ & $<0.001$ & 3.137 & $1.674-5.879$ & $<0.001$ \\
\hline & 2.174 & $1.431-3.302$ & $<0.001$ & 1.661 & $1.074-2.568$ & 0.023 \\
\hline & 1.073 & $0.690-1.668$ & 0.755 & & & \\
\hline & 1.763 & $0.852-3.649$ & 0.126 & & & \\
\hline & 1.058 & $0.671-1.668$ & 0.808 & & & \\
\hline & 0.898 & $0.596-1.355$ & 0.610 & & & \\
\hline & 2.509 & $1.637-3.846$ & $<0.001$ & 2.130 & $1.370-3.312$ & 0.001 \\
\hline
\end{tabular}

indicate that DKK1 plays a tumor suppressor role in GC stem cells. However, the authors only evaluated the $\mathrm{CD}_{4} 4^{+} \mathrm{GC}$ cell line. Although cancer stem cells play a crucial role in GC, they are not entirely representative of GC. Given that the tumor cell, tumor environment, and cell-cell interactions all contribute to GC, studies using limited gastric cancer cell line was further validated.

Activation of Wnt/ $\beta$-catenin is found in $30 \%$ to $50 \%$ of GC tissue samples and cell lines [6, 24]. Furthermore, recent studies that used high-throughput sequencing methods showed that CTNNB1, which encodes $\beta$-catenin, is a driver gene for GC [25-27]. However, CTNNB1 gene mutations were only detected in $4 \%$ to $9 \%$ of sporadic GC tumors $[28,29]$. These findings suggest that aberrant activation of Wnt signaling pathway is modulated mainly by Wnt ligands and negative regulators, not through mutation of the CTTNB1 gene. In this study, high DKK1 expression was significantly related to positive $\beta$-catenin expression in AGC samples. Given that DKK1 inhibits $\beta$-catenin, this finding may seem contradictory. However, several tumor studies revealed that high DKK1 expression is correlated with activation of the $\mathrm{Wnt} / \beta$-catenin pathway in both hepatocellular carcinoma and hilar cholangiocarcinoma $[20,30]$. These findings could be explained by a disruption in the negative feedback loop between DKK1 and the Wnt/ $\beta$-catenin pathway, which could result from a high level of secreted DKK1 [31]. In addition, DKK1 is also a downstream target gene of $\beta$-catenin/TCF, which is a direct target of activated $\beta$-catenin [32].

The OS and DFS of patients with high DKK1 and negative $\beta$-catenin expression were not different from those with high DKK1 and positive $\beta$-catenin expression. These results suggest that the effect of high DKK1 expression in AGC could be an independent of $\beta$-catenin status. Conversely, high DKK1 expression, which does not affect canonical Wnt/ $\beta$-catenin signaling, is still a prognostic factor for patients with AGC.

Our results suggest that high DKK1 expression affects prognosis regardless of $\beta$-catenin activation. Several previous studies showed that DKK1 promotes malignancy via non-canonical Wnt pathway mechanisms. In hepatocellular carcinoma, high DKK1 mRNA and protein expression was correlated with poor OS and DFS. In addition, a positive relationship among DKK1 expression, JNK phosphorylation, and RhoA levels was identified [33]. These results indicate that the malignant potential can be increased by the interaction between DKK1 and the non-canonical Wnt pathway, which consists of the Wnt/Ca ${ }^{2+}$ and Wnt/PCP pathways and does not involve activation of $\beta$-catenin [34]. Moreover, Kimura et al. reported that cytoskeleton-associated 
Table 5 Univariate and multivariate analyses of disease-free survival and overall survival using the Cox proportional hazards model for all patients

\begin{tabular}{|c|c|c|c|c|c|c|}
\hline \multirow[t]{2}{*}{ Characteristics } & \multicolumn{3}{|c|}{ Univariate analysis of DFS } & \multicolumn{3}{|c|}{ Multivariate analysis of DFS } \\
\hline & Hazard ratio & $95 \% \mathrm{Cl}$ & $p$-value & Hazard ratio & $95 \% \mathrm{Cl}$ & $p$-value \\
\hline Age (> 62 vs. $\leq 62)$ & 1.251 & $0.725-2.158$ & 0.420 & & & \\
\hline Sex (female vs male) & 1.005 & $0.544-1.856$ & 0.987 & & & \\
\hline Advanced T stage (pT4 vs. pT2/3) & 7.564 & $2.356-24.286$ & 0.001 & 6.569 & $2.014-21.422$ & $<0.001$ \\
\hline Advanced N stage (pN2/3 vs. pNo/1) & 3.544 & $1.959-6.409$ & $<0.001$ & 2.500 & $1.359-4.601$ & 0.003 \\
\hline Lauren's classification (diffuse vs. non-diffuse) & 1.255 & $0.680-2.317$ & 0.467 & & & \\
\hline Venous invasion (yes vs. no) & 1.940 & $0.771-4.883$ & 0.159 & & & \\
\hline Tumor grade (poor vs well to moderate) & 1.172 & $0.626-2.197$ & 0.619 & & & \\
\hline Adjuvant chemotherapy (yes vs. no) & 1.196 & $0.687-2.083$ & 0.526 & & & \\
\hline \multirow{11}{*}{$\begin{array}{l}\text { High DKK1 and positive ß-catenin } \\
\text { (yes vs. no) }\end{array}$} & 2.512 & $1.430-4.413$ & 0.001 & 2.357 & $1.291-4.306$ & 0.005 \\
\hline & \multicolumn{3}{|c|}{ Univariate analysis of OS } & \multicolumn{3}{|c|}{ Multivariate analysis of OS } \\
\hline & 1.934 & $1.269-2.946$ & 0.002 & 1.820 & $1.189-2.785$ & 0.006 \\
\hline & 0.656 & $0.403-1.069$ & 0.091 & & & \\
\hline & 3.356 & $1.825-6.170$ & $<0.001$ & 3.137 & $1.674-5.879$ & $<0.001$ \\
\hline & 2.174 & $1.431-3.302$ & $<0.001$ & 1.661 & $1.074-2.568$ & 0.023 \\
\hline & 1.073 & $0.690-1.668$ & 0.755 & & & \\
\hline & 1.763 & $0.852-3.649$ & 0.126 & & & \\
\hline & 1.058 & $0.671-1.668$ & 0.808 & & & \\
\hline & 0.898 & $0.596-1.355$ & 0.610 & & & \\
\hline & 2.522 & $1.645-3.867$ & $<0.001$ & 2.140 & $1.343-3.409$ & 0.001 \\
\hline
\end{tabular}

protein 4, a receptor for DKK1, mediates DKK1 signaling to promote cancer cell proliferation via the PI3K/ AKT pathway and was associated with an unfavorable prognosis in pancreatic and lung cancer patients [35]. Together, these results suggest that high DKK1 expression acts through $\beta$-catenin-independent mechanisms to increase the malignant potential and decrease survival in patients with AGC.

Unfortunately, molecular targeting therapies for AGC are limited to trastuzumab and ramucirumab [36, 37]. Due to the shortage of promising target agents for GC, new targets molecules with potential agents are urgently needed. The efficacy of an anti-DKK1 antibody has been investigated in multiple myeloma and prostate cancers that were associated with bone resorption [38, 39]. However, further preclinical studies to determine the effectiveness of anti-DKK1 antibody in GC are required.

\section{Conclusions}

We found that high DKK1 expression was correlated with a positive $\beta$-catenin status. In addition, patients with high DKK1 expression who were positive for $\beta$ catenin had a poor prognosis. However, patients with high DKK1 expression who were negative for $\beta$-catenin also demonstrated a poor prognosis. In the multivariate analysis, high DKK1 expression only or high DKK1 expression with $\beta$-catenin positivity were an independent prognostic factor for OS and DFS in patients with AGC. Together, these results suggest that DKK1 may act as a biomarker and therapeutic target in AGC.

\section{Additional file}

Additional file 1: Table S1. Correlation between clinicopathogic

findings and $ß$-cateinin expression. (DOCX $21 \mathrm{~kb}$ )

\section{Abbreviations}

AGC: Advanced gastric cancer; Cl: Confidence interval; DFS: Disease-free survival; DKK1: Dickkopf-1; EGC: Early gastric cancer; FZ: Frizzled receptor; GC: Gastric cancer; HR: Hazard ratio; LRP: Lipoprotein receptor-related protein; OS: Overall survival; TCF: T-cell factor

\section{Funding}

This study was supported by Basic Science Research Program through the National Research Foundation of Korea (NRF) funded by the Ministry of Science, ICT \& Future Planning (NRF-2015R1C1A1A01054591) (Y. H. K.), and by The Catholic University of Korea Uijeongbu St. Mary's Hospital Clinical Research Laboratory Foundation made in the program year of 2012. (Y. H. K.) The funding bodies had no role in the design of the study and collection, analysis, and interpretation of data and in the writing of the manuscript.

Availability of data and materials

Additional data and materials may be obtained from the corresponding author on reasonable request. 


\section{Authors' contributions}

SAH conceptualization, data collection, formal analysis, investigation, methodology, writing the original draft, review and editing. SHY formal analysis, investigation, methodology, writing the original draft, review and editing. HHL, DSS, HSW data collection, review and editing the manuscript. OK investigation, methodology, writing the original draft. YHK Conceptualization, funding acquisition, investigation, project administration, resources, supervision, writing the original draft, and review and editing. All authors read and approved the manuscript.

\section{Authors' information}

Soon Auck Hong: Clinical Assistant Professor, Department of Pathology, Soonchunhyang Cheonan University Hospital, Cheonan, Republic of Korea.

Soo Hyun Yoo: Pathologist, Medical Clinic Laboratory Department of U2Bio Co. Ltd., Seoul, Republic of Korea.

Han Hong Lee: Associate Professor, Department of General Surgery, College of Medicine, The Catholic University of Korea, Seoul, Republic of Korea.

Der Sheng Sun: Clinical Associate Professor, Division of Oncology, Department of Internal Medicine, College of Medicine, The Catholic University of Korea, Seoul, Republic of Korea.

Hye Sung Won: Associate Professor, Division of Oncology, Department of Internal Medicine, College of Medicine, The Catholic University of Korea, Seoul, Republic of Korea.

Okran Kim: Researcher, Cancer Research Institute, College of Medicine, The Catholic University of Korea, Seoul, Republic of Korea.

Yoon Ho Ko: Division of Oncology, Department of Internal Medicine, College of Medicine, The Catholic University of Korea, Seoul, Republic of Korea and Cancer Research Institute, College of Medicine, The Catholic University of Korea, Seoul, Republic of Korea.

\section{Ethics approval and consent to participate}

This study was approved by the Institutional Research Ethics Board of Uijeongbu St. Mary's Hospital of the Catholic University of Korea. Due to the retrospective nature of the study and the fact that no identifiable information is included, informed consent was not required according to laws of the Institutional Research Ethics Board of Uijeongbu St. Mary's Hospital of the Catholic University of Korea. The project was compliance with the Helsinki Declaration.

\section{Competing interests}

The authors declare that they have no competing interests.

\section{Publisher's Note}

Springer Nature remains neutral with regard to jurisdictional claims in published maps and institutional affiliations.

\section{Author details \\ 'Department of Pathology, Soonchunhyang University Cheonan Hosptial, Cheonan, Republic of Korea. ${ }^{2}$ Medical Clinic Laboratory Department of U2Bio Co. Ltd., Seoul, Republic of Korea. ${ }^{3}$ Department of General Surgery, College of Medicine, The Catholic University of Korea, Seoul, Republic of Korea. ${ }^{4}$ Division of Oncology, Department of Internal Medicine, College of Medicine, The Catholic University of Korea, 222, Banpodaero, Seochogu, Seoul 06591, Republic of Korea. ${ }^{5}$ Cancer Research Institute, College of Medicine, The Catholic University of Korea, Seoul, Republic of Korea.}

Received: 15 November 2017 Accepted: 22 April 2018

Published online: 02 May 2018

\section{References}

1. Ferlay J, Soerjomataram I, Dikshit R, Eser S, Mathers C, Rebelo M, Parkin DM, Forman D, Bray F. Cancer incidence and mortality worldwide: sources, methods and major patterns in GLOBOCAN 2012. Int J Cancer. 2015;136(5):E359-86.

2. Yada T, Yokoi C, Uemura N. The current state of diagnosis and treatment for early gastric cancer. Diagn Ther Endosc. 2013;2013:241320.

3. Wagner AD, Grothe W, Haerting J, Kleber G, Grothey A, Fleig WE. Chemotherapy in advanced gastric cancer: a systematic review and metaanalysis based on aggregate data. J Clin Oncol. 2006;24(18):2903-9.
4. MacDonald BT, Tamai K, He X. Wnt/beta-catenin signaling: components, mechanisms, and diseases. Dev Cell. 2009;17(1):9-26.

5. Zhan T, Rindtorff N, Boutros M. Wnt signaling in cancer. Oncogene. 2017; 36(11);1461-73.

6. Clements WM, Wang J, Sarnaik A, Kim OJ, MacDonald J, Fenoglio-Preiser C, Groden J, Lowy AM. $\beta$-catenin mutation is a frequent cause of Wnt pathway activation in gastric cancer. Cancer Res. 2002;62(12):3503-6.

7. Ikenoue T, ljichi H, Kato N, Kanai F, Masaki T, Rengifo W, Okamoto M, Matsumura M, Kawabe T, Shiratori Y. Analysis of the $\beta$-catenin/T cell factor signaling pathway in 36 gastrointestinal and liver Cancer cells. Jpn J Cancer Res. 2002;93(11):1213-20

8. Anastas JN, Moon RT. WNT signalling pathways as therapeutic targets in cancer. Nat Rev Cancer. 2013:13(1):11-26.

9. Cruciat $C M$, Niehrs C. Secreted and transmembrane wnt inhibitors and activators. Cold Spring Harb Perspect Biol. 2013;5(3):a015081.

10. Mao B, Wu W, Davidson G, Marhold J, Li M, Mechler BM, Delius H, Hoppe D, Stannek $P$, Walter $C$. Kremen proteins are Dickkopf receptors that regulate Wnt/B-catenin signalling. Nature. 2002;417(6889):664-7.

11. Bafico A, Liu G, Yaniv A, Gazit A, Aaronson SA. Novel mechanism of Wnt signalling inhibition mediated by Dickkopf-1 interaction with LRP6/arrow. Nat Cell Biol. 2001;3(7):683-6.

12. Mao B, Wu W, Li Y, Hoppe D, Stannek P, Glinka A, Niehrs C. LDL-receptor-related protein 6 is a receptor for Dickkopf proteins. Nature. 2001:411(6835):321-5.

13. Menezes ME, Devine DJ, Shevde LA, Samant RS. Dickkopf1: a tumor suppressor or metastasis promoter? Int J Cancer. 2012;130(7):1477-83.

14. Lee HS, Lee HE, Park DJ, Kim HH, Kim WH, Park KU. Clinical significance of serum and tissue Dickkopf-1 levels in patients with gastric cancer. Clin Chim Acta. 2012;413(21-22):1753-60

15. Gao C, Xie R, Ren C, Yang X. Dickkopf-1 expression is a novel prognostic marker for gastric cancer. J Biomed Biotechnol. 2012;2012:804592.

16. Wang B, Liu J, Ma LN, Xiao HL, Wang YZ, Li Y, Wang Z, Fan L, Lan C, Yang M. Chimeric 5/35 adenovirus-mediated Dickkopf-1 overexpression suppressed tumorigenicity of CD44+ gastric cancer cells via attenuating Wnt signaling. J Gastroenterol. 2013:48(7):798-808.

17. Jia X, Li N, Peng C, Deng Y, Wang J, Deng M, Lu M, Yin J, Zheng G, Liu H. miR-493 mediated DKK1 down-regulation confers proliferation, invasion and chemo-resistance in gastric cancer cells. Oncotarget. 2016; 7(6):7044.

18. Kim B, Byun SJ, Kim YA, Kim JE, Lee BL, Kim WH, Chang MS. Cell cycle regulators, APC/beta-catenin, NF-kappaB and Epstein-Barr virus in gastric carcinomas. Pathology. 2010;42(1):58-65.

19. Wirths O, Waha A, Weggen S, Schirmacher P, Kühne T, Goodyer CG, Albrecht S, von Schweinitz D, Pietsch T. Overexpression of human Dickkopf1, an antagonist of wingless/WNT signaling, in human hepatoblastomas and Wilms' tumors. Lab Investig. 2003;83(3):429-34.

20. Chen L, Li M, Li Q, Wang C-j, Xie S-q. DKK1 promotes hepatocellular carcinoma cell migration and invasion through $\beta$-catenin/MMP7 signaling pathway. Mol Cancer. 2013;12(1):1.

21. Voorzanger-Rousselot N, Goehrig D, Journe F, Doriath V, Body JJ, Clezardin P, Garnero P. Increased Dickkopf-1 expression in breast cancer bone metastases. Br J Cancer. 2007;97(7):964-70.

22. Tian E, Zhan F, Walker R, Rasmussen E, Ma Y, Barlogie B, Shaughnessy JD Jr. The role of the Wnt-signaling antagonist DKK1 in the development of osteolytic lesions in multiple myeloma. N Engl J Med. 2003;349(26):2483-94.

23. Orditura M, Galizia G, Sforza V, Gambardella V, Fabozzi A, Laterza MM, Andreozzi F, Ventriglia J, Savastano B, Mabilia A, et al. Treatment of gastric cancer. World J Gastroenterol. 2014;20(7):1635-49.

24. Ooi CH, Ivanova T, Wu J, Lee M, Tan IB, Tao J, Ward L, Koo JH, Gopalakrishnan V, Zhu Y. Oncogenic pathway combinations predict clinical prognosis in gastric cancer. PLoS Genet. 2009;5(10):e1000676.

25. Lee J, van Hummelen P, Go C, Palescandolo E, Jang J, Park HY, Kang SY, Park JO, Kang WK, MacConaill L, et al. High-throughput mutation profiling identifies frequent somatic mutations in advanced gastric adenocarcinoma. PLoS One. 2012;7(6):e38892.

26. Holbrook JD, Parker JS, Gallagher KT, Halsey WS, Hughes AM, Weigman VJ, Lebowitz PF, Kumar R. Deep sequencing of gastric carcinoma reveals somatic mutations relevant to personalized medicine. J Transl Med. 2011;9(1):119.

27. Wang K, Yuen ST, XU J, Lee SP, Yan HH, Shi ST, Siu HC, Deng S, Chu KM, Law $\mathrm{S}$, et al. Whole-genome sequencing and comprehensive molecular profiling identify new driver mutations in gastric cancer. Nat Genet. 2014; 46(6):573-82. 
28. Wang K, Kan J, Yuen ST, Shi ST, Chu KM, Law S, Chan TL, Kan Z, Chan AS, Tsui WY, et al. Exome sequencing identifies frequent mutation of ARID1A in molecular subtypes of gastric cancer. Nat Genet. 2011;43(12):1219-23.

29. Cancer Genome Atlas Research N. Comprehensive molecular characterization of gastric adenocarcinoma. Nature. 2014;513(7517):202-9.

30. Shi XD, Yu XH, Wu WR, Xu XL, Wang JY, Xu LB, Zhang R, Liu C. Dickkopf-1 expression is associated with tumorigenity and lymphatic metastasis in human hilar cholangiocarcinoma. Oncotarget. 2016;7(43):70378-87.

31. Gonzalez-Sancho JM, Aguilera O, Garcia JM, Pendas-Franco N, Pena C, Cal S, Garcia de Herreros A, Bonilla F, Munoz A. The Wnt antagonist DICKKOPF-1 gene is a downstream target of beta-catenin/TCF and is downregulated in human colon cancer. Oncogene. 2005;24(6):1098-103.

32. Niida A, Hiroko T, Kasai M, Furukawa Y, Nakamura Y, Suzuki Y, Sugano S, Akiyama T. DKK1, a negative regulator of Wnt signaling, is a target of the [beta]-catenin/TCF pathway. Oncogene. 2004;23(52):8520.

33. Tao YM, Liu Z, Liu HL. Dickkopf-1 (DKK1) promotes invasion and metastasis of hepatocellular carcinoma. Digestive and liver disease : official journal of the Italian Society of Gastroenterology and the Italian Association for the Study of the Liver. 2013;45(3):251-7.

34. Kikuchi A, Yamamoto $H$, Sato A, Matsumoto S. New insights into the mechanism of Wnt signaling pathway activation. Int Rev Cell Mol Biol. 2011; 291(21):e71.

35. Kimura H, Fumoto K, Shojima K, Nojima S, Osugi Y, Tomihara H, Eguchi H, Shintani $Y$, Endo $H$, Inoue M, et al. CKAP4 is a Dickkopf1 receptor and is involved in tumor progression. J Clin Invest. 2016;126(7):2689-705.

36. Apicella M, Corso S, Giordano S. Targeted therapies for gastric cancer: failures and hopes from clinical trials. Oncotarget. 2017;

37. Fuchs CS, Tomasek J, Yong CJ, Dumitru F, Passalacqua R, Goswami C, Safran H, dos Santos LV, Aprile G, Ferry DR, et al. Ramucirumab monotherapy for previously treated advanced gastric or gastro-oesophageal junction adenocarcinoma (REGARD): an international, randomised, multicentre, placebo-controlled, phase 3 trial. Lancet. 2014;383(9911):31-9.

38. Fulciniti M, Tassone P, Hideshima T, Vallet S, Nanjappa P, Ettenberg SA, Shen Z, Patel N, Tai YT, Chauhan D, et al. Anti-DKK1 mAb (BHQ880) as a potential therapeutic agent for multiple myeloma. Blood. 2009;114(2):371-9.

39. Hall CL, Bafico A, Dai J, Aaronson SA, Keller ET. Prostate cancer cells promote osteoblastic bone metastases through Wnts. Cancer Res. 2005; 65(17):7554-60.

\section{Ready to submit your research? Choose BMC and benefit from:}

- fast, convenient online submission

- thorough peer review by experienced researchers in your field

- rapid publication on acceptance

- support for research data, including large and complex data types

- gold Open Access which fosters wider collaboration and increased citations - maximum visibility for your research: over $100 \mathrm{M}$ website views per year

At BMC, research is always in progress.

Learn more biomedcentral.com/submissions 